\title{
The Research on the control system of the autonomous vehicle for sea floor prospecting with chaotic optimization theory
}

\author{
Zou En ${ }^{1,2}$, Huo Qing ${ }^{2}$,Huang Shuihong ${ }^{2}$,Chen Shengchuang ${ }^{1}$
}

1. College of zhujiang. South China Agricultural University. Guangzhou 510900, P.R.China.

2.College of Engineer. South China Agricultural University. Guangzhou 510642, P.R.China.

\begin{abstract}
In order to ensure the accuracy and precision about the trace of the autonomous vehicle for the sea floor prospecting, double- ring, series -connection closed-loop control mode is used in the paper. Fuzzy controller is used for controlling velocity in the inner-ring, and fuzzy neutral network controller is used in the out ring. For optimizing the control performance, chaotic algorithm is used to optimize the parameters in the fuzzy controller and the structure, weights and membership function in the fuzzy neural network controller. The experimental results show: Quick response speed, better self-adaptive performance and higher control quality are obtained in the system.
\end{abstract}

\section{Introduction}

With the increase of the population, resources are need much more for human beings. Despite that there are so many kinds of resources which have been discovered in the continent, the continental resources decreased quickly, and cannot satisfied the demand from human beings. The scientists have proven that there are so rich kinds of resources which exist in the deep ocean, so people gradually look for those resources in the deep ocean.

Many mental ores are located in the surface of sea floor which range from minus $3 \mathrm{~km}$ to 6 $\mathrm{km}$. the pressure in the minus $6 \mathrm{~km}$ sea floor is up to 500 atmospheric pressures. People cannot operate directly in the deep sea floor for those ores. Hence the research on the autonomous vehicle for sea floor prospecting which used for substituting human is appeared.

Considering the complexity which the autonomous vehicle will meet in the sea floor, those factors are included: variable geological conditions, the ocean flow from different cross layer, and random motions from transportation tubes. These interferences are non-linear, unpredictable and random variable. The normal control algorithm cannot be realized in the complex control process. Fuzzy control is a non-mode control method based on knowledge, and no need for precise mathematical mode. It can be applied in complicated industrial control with good robust performance.

The control theme for the autonomous vehicle for sea floor prospecting is based on position setting and race tracing. A double -ring, series -connection closed-loop control system is used for ensuring the quickness and precision to the race tracing. Fuzzy neural network is used to control the position of the autonomous vehicle in the out ring. Fuzzy controller is used to control the velocity of the autonomous vehicle in the inner ring. At the same time, according to the characteristic of the chaotic variables, the structure and the parameters in the fuzzy neural network and the parameters in the fuzzy controller are searched and optimized with chaotic optimization theory in the system, the optimized efficiency and performance are improved.

\section{2. the motion equation for the autonomous vehicle for sea floor prospecting}

Limited by the sea floor environments and the characteristics of the vehicle, the speed of the vehicle is not so quick as imagined. The max speed is $1 \mathrm{~m} / \mathrm{s}$. the structure of the wheel is caterpillar for its working environment is located in the minus $600 \mathrm{~m}-6000 \mathrm{~m}$ sea floor. Doubles wheels driven mode is used with front wheel directing, and independent back wheel. The motion path is controlled by means of controlling the velocity difference between the right wheel and left wheel. The motion equation is given: 
Considering the two-dimentional motion mode, when the vehicle changes direction horizontally the forward speed for its center can be expressed as follows:

$$
v=\frac{v_{L}+v_{R}}{2}
$$

In this expression, $v_{L}$ and $v_{R}$ are the speed for the left and right wheel respectively.

The angular velocity for the vehicle's changing direction can be expressed approximetly

$$
\theta=\frac{v_{L}+v_{R}}{b}
$$

$\mathrm{b}$ is distance of the belts center.

So the speed for the left and right wheel can be obtained as:

$$
v_{L}=v+\frac{\theta A}{2}, \quad v_{R}=v-\frac{\theta A}{2}
$$

The $\mathrm{x}, \mathrm{y}$ coordinates for the mass center of the vehicle are

$$
v_{L}=v+\frac{\theta A}{2}, v_{R}=v-\frac{\theta A}{2}
$$

The real revolving speed can be measured by the velocity encoder.

\section{The design to the control system of the autonomous vehicle for sea floor prospecting}

\section{1 the structure and its characteristic about the control system}

For ensuring the quickness and precision about the race tracing, double-ring, series-connection is used in the control system. Considering the non-linear, time-variable and indeterminate, satisfying the high quality demand in the dynamic process from the system. Fuzzy controller and fuzzy neural network controller are used respectively. The structure is shown as Fig.1

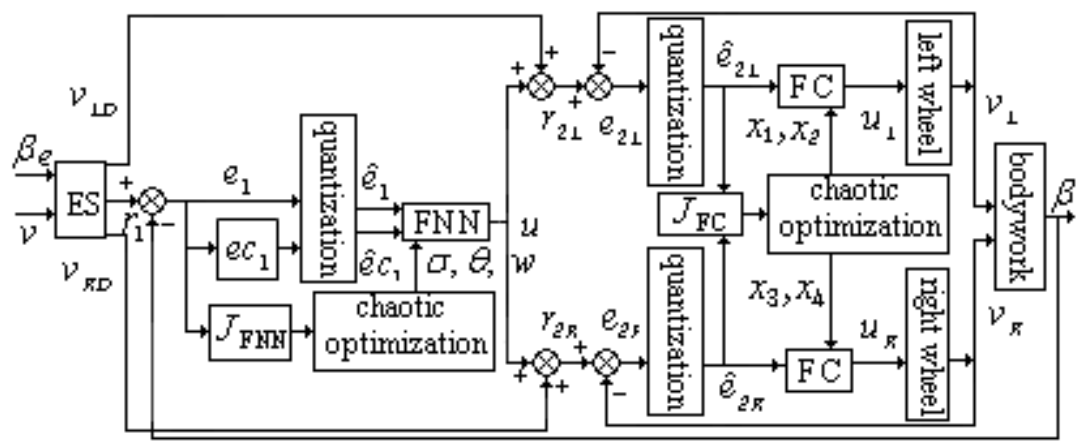

Fig. 1 the schematic diagram for the autonomous vehicle for sea floor prospecting

$\beta_{e}$ is the position setting; $v$ is the velocity setting; $r_{1}=\beta_{e}$ is the out ring position setting; $v_{L D}$ is velocity setting of the left wheel; $v_{R D}$ is velocity setting of the right wheel; $r_{2 L}$ is left wheel setting of the inner ring; $r_{2 R}$ is right wheel setting of the inner ring; $v_{L}$ is the output of the left wheel ; $v_{R}$ is the output of the right wheel; $\beta$ is the output of the position.

In the running of the system, quick response is needed to the velocity control, so the inner ring is set to be a velocity ring and the out ring is set to be a position ring. The forward path of the vehicle is controlled by controlling the angle of the position. The output of the position ring can correct the the velocity setting of the left, right wheels. So the deviation of the position angle can change the velocity setting of the left, right wheels. As a result , the moving direction of the vehicle can be changed in time, and the path control can be realized

\section{2 the design on the fuzzy controller}

Fuzzy controller is used as in reference [5], the membership functions of its input $\hat{e}_{2}$ and output $u$ are triangle functions. The membership functions of $\hat{e}_{2}$ is fixed and the parameters $x_{1} 、 x_{2} \quad\left(x_{1}\right.$ and $x_{2}$ are structure parameters of controller) of the membership functions of output $u$ is adjustable, shown in Fig.2. 

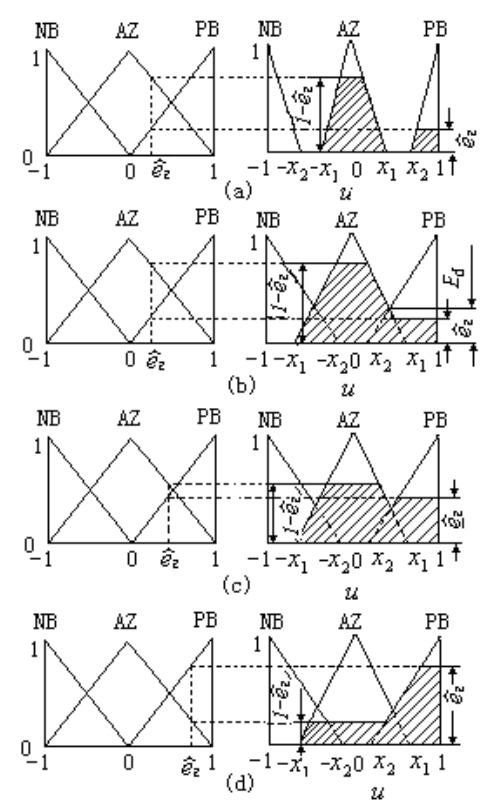

Fig.2 Membership function of fuzzy controller

In the Fig. $2, e_{d}=\left(x_{1}-x_{2}\right) /\left(1+x_{1}-x_{2}\right)$, the shadow area is the fuzzy output value when the parameters $x_{1} 、 x_{2}$ and the deviation $\hat{e}_{2}$ are taken a different values. Assume $e_{1}=1-\left|\hat{e}_{2}\right|$, $e_{2}=1+\left|\hat{e}_{2}\right|, \quad e_{3}=1-2\left|\hat{e}_{2}\right|, \quad e_{4}=x_{1}-x_{2}, \quad e_{5}=1-x_{2}, \quad$ the algorithm of the fuzzy controller is

(1)If $0<x_{1} \leq x_{2}<1$,

$$
u=\frac{e_{5} \hat{e}_{2}\left[3 x_{2}\left(2-\left|\hat{e}_{2}\right|\right)+e_{5}\left(3-\hat{e}_{2}^{2}\right)\right]}{3\left[2 x_{1}\left(1-\hat{e}_{2}^{2}\right)+e_{5}\left(2\left|\hat{e}_{2}\right|-\hat{e}_{2}^{2}\right)\right]}
$$

The result can shown in (a) of the Fig. 2

(2)If $0<x_{2}<x_{1}<1$, there will be three cases

(1) $0 \leq\left|\hat{e}_{2}\right| \leq e_{d}$

$$
u=\frac{\hat{e}_{2}\left[3\left(1-x_{1}^{2}\right)+3 x_{1}^{2}\left|\hat{e}_{2}\right|-x_{1}^{2} \hat{e}_{2}^{2}\right]}{3\left[2 x_{1}+2\left(1-x_{1}\right)|\hat{e}|-x_{1} \hat{e}_{2}^{2}\right]}
$$

The result can shown in (b) of the Fig.2

(2) $e_{d}<\left|\hat{e}_{2}\right|<1-e_{d}$,

$$
u=\frac{\hat{e}_{2}\left\{e_{5}\left|\hat{e}_{2}\right|\left[3 x_{2}\left(2-\left|\hat{e}_{2}\right|\right)+e_{5}\left(3-\hat{e}_{2}^{2}\right)\right]-e_{4} e_{d}\left[\left(3-e_{d}\right) x_{1}-e_{4}\right]\right\}}{3\left|\hat{e}_{2}\right|\left[2 x_{1}\left(1-\hat{e}_{2}^{2}\right)+e_{5}\left(2\left|\hat{e}_{2}\right|-\hat{e}_{2}^{2}\right)-e_{4} e_{d}\right]}
$$

The result can shown in (c) of the Fig.2

(3) $\quad 1-e_{d} \leq\left|\hat{e}_{2}\right| \leq 1$,

$$
u=\frac{\hat{e}_{2}\left\{e_{4}\left[3-x_{1}^{2}\left(1+\left|\hat{e}_{2}\right|+\hat{e}_{2}^{2}\right)\right]-e_{5} e_{3}\left[3-e_{5}\left(1-\left|\hat{e}_{2}\right|+\hat{e}_{2}^{2}\right)\right]\right\}}{3\left|\hat{e}_{2}\right|\left[e_{1}\left(2+x_{1} e_{2}\right)-e_{5} e_{3}\right]}
$$

The result can shown in (d) of the Fig. 2

\subsection{The design on the fuzzy neural network controller}

The two- input, one -output and four layers of forward network, and four - subset are used in the fuzzy neural network. The structure of the network is 4-8-16-1.the outputs from the first layer to the fourth layer are

$$
O_{i}^{(1)}=x_{i}=\hat{e}_{1} \text { or } \hat{e} c_{1}, \quad x_{i} \in[-1,1], \quad i=1,2
$$




$$
O_{j}^{(2)}=\mu_{F_{i j}}=\exp \left[-\frac{\left(x_{i}-\theta_{i j}\right)^{2}}{\sigma_{i j}^{2}}\right], \quad j=1,2,3,4
$$

In the expression, $\theta, \sigma$ are the center and width of the Gauss membership function respectively.

$$
\begin{aligned}
& O_{k}^{(3)}=\prod_{i=1}^{2} w_{j k}^{(3)} O_{j}^{(2)}, \quad k=1,2, \cdots, 16 \\
& O^{(4)}=\widehat{u}=\frac{\sum_{l} w_{k l}^{(4)} O_{k}^{(3)}}{\sum_{l} O_{k}^{(3)}}, \quad l=1
\end{aligned}
$$

\section{The design on the controller based on chaotic optimization ${ }^{[6-7]}$}

The basic idea in the paper is that the variables in the chaotic state are put into the parameters in the controller. By means of utilizing the ergodic property, randomization and regularity, the parameters of the fuzzy controller, the structure, weights and membership functions of the fuzzy neural network controller are optimized. According to Logistic mapping

$$
q_{n+1}=\mu \mathrm{q}_{n}\left(1-q_{n}\right) \quad n=0,1,2, \cdots N \quad q_{0} \in(0,1)
$$

where $q=\left(q_{1, n}, q_{2, n}, \cdots, q_{L, n}\right)$ are the number of chaotic variables, $N$ is the frequency of chaos learning, $\mu$ is control parameter. When $\mu=4$, the mapping is perfect mapping of $(0,1)$ interval and system gets into in full chaos state, $q_{n}$ are ergodic in $(0,1)$ interval and generate chaotic sequence. Since chaotic state is extreme sensitiveness to initial value, to fetch some initial values with tiny discrepancy to equation (13) can get a sequence of chaotic variables $\left\{q_{n}\right\}$ with different traces.

\subsection{The fuzzy controller with velocity ring}

The out ring is break when the inner ring is optimized. The velocity of the wheel is controlled with inner ring. Because the controllers of the right and left wheel are the same, the same parameters are the same in the two fuzzy controllers. Here left wheel is taken as an example, its parameters are

$$
J_{F C}=\min \left[\alpha \sum_{T} T^{2}\left|e_{2 L}(T)\right| / \max \left(e_{2 L}(T)\right)\right]
$$

Weights $\alpha=0.5, \quad \min J_{F C}=10^{-3}$, the optimized variables are two parameters $x_{i}, i=1,2$ in the fuzzy controller the steps of the optimization are

Step1 To fetch two different initial random values in $(0,1)$ interval and substitute in Logistic mapping of equation (13) respectively, two chaos variables are obtained

Step2 The chaos variables are substituted into equation(5)-(8) respectively, according to the equation (14) calculate the corresponding object function $J_{F C}$.

Step3 If the condition is satisfied, the search process is terminated and turned in to step4. Otherwise return to step2.

Step4 Finding the minimum value in the system, optimizing the corresponding value of the fuzzy controller, and saving it.

The velocity system is experimented after the values are set in the fuzzy controller. The vehicle is observed whether it can move with direct path. The experimental results show that the vehicle can move along the direct path basically. The design on the fuzzy controller with chaotic theory is reasonable. So those parameters are input to the closed-loop system as the given values in the fuzzy controller.

\subsection{The fuzzy neural network controller with position ring}

For getting a better fuzzy neural network, the structure of it should be simple with satisfying the performance. But the control precision will be low if there are less nodes. Considering the 
precision and the simplicity of the structure, the parameters of the performance are

$$
\begin{aligned}
& J_{F N N}=\min (E+\lambda C) \\
& \text { Where } \quad E=\frac{1}{2} \sum_{t=1}^{N}(r-y) \\
& \text { and } \quad C=\sum_{k=1}^{F} \frac{\left(w_{k} / \alpha\right)}{1+\left(w_{k} / \alpha\right)}
\end{aligned}
$$

This item is use to control the complexity in the network. $w_{k}$ is the linkage weights between the third and the fourth layer. The constant $\alpha=0.02$, the modulating factor $\lambda=0.2$, $J_{\text {FNN }}=10^{-3}$.

The parameters of FNN will be optimized after modification of the centers and widths of Layer (2) and the link weights of Layer(3) to (4). therefore, the total number of parameters needing to be optimized should be: $2 * 4+16=24$. Because the linkage weights values of the regular nodes between layer 3 and 4 are corresponding. Its values represent the intense of the corresponding regularity. So the following method for optimizing weights values is that the 24 different initial values are picked at random in $(0,1)$ interval and are substituted in the Logistic mapping of equation(8) and can get 24 traces different chaos variable sequence. In order that each chaos variable can map to itself interval, mapping of chaos variables can be described as follows:

$$
\begin{array}{lc}
q_{i, n}=2 x_{i, n}-1 & (i=1,2, \cdots, 20) \\
q_{j, n}=x_{i, n} & (j=1,2,3,4)
\end{array}
$$

where $q_{i, n}$ are parameters of membership function's weightss and center, the equation (18) can ensure $-1<(w, \theta)<1, \quad q_{j n}$ are parameters of membership function's width, the equation (19) can ensure $0<\sigma<1$.

The preceding chaotic variables are put into the expression (9)-(12) forsearching the optimized value. $w_{k}$ is compared with the constant $\alpha$ according to the expression (15)-(17), then computing the performance value $J_{F N N}$. If the result is $\left|w_{k}\right| \leq \alpha, k=1,2, \cdots, 16$, it means the effect is so tiny that it enabling the corresponding $O_{k}^{(3)}=0$, eliminating this regular node, and its linkage between input and output. The whole optimized solutions with optimized structure can be obtained until the performance demand is satisfied.

\section{The experimental results about the autonomous vehicle for sea floor prospecting}

After being optimized by off-line chaotic optimization, the structure and parameters of fuzzy controller and fuzzy neutral controller are improved. By means of fixing those values, modulating the weights values of the fuzzy neutral network on-line to the given path and position signal, the autonomous vehicle for sea floor prospecting is experimented and adjusted finally. The path diagram about the experiment is shown in Fig.3. The solid line ( red line) is the ideal path, and the broken line (green line) is the real path. The control results show that the autonomous vehicle for sea floor prospecting can move along the real path with high precision control system and quick response 


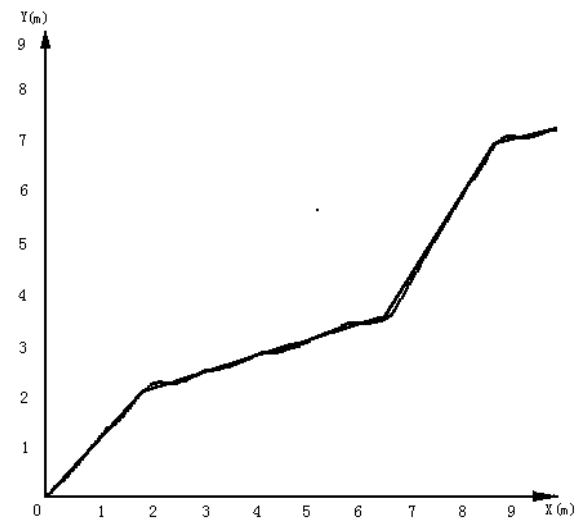

Fig. 3 the control result about the path of the autonomous vehicle for sea floor prospecting

\section{Conclusion}

A double- ring, series -connection closed-loop control system has been adopted in the paper to the intelligent autonomous vehicle for sea floor prospecting. Fuzzy controller is used for controlling velocity in the inner-ring, and fuzzy neutral network controller is used in the out ring. The design to the whole control system is that parameters in the fuzzy controller, structure, weights values and membership functions in the fuzzy neutral controller are obtained with chaotic optimization off-line. The final experiment is good as imagined. The problems about the real-time control are solved with better effect. The method for chaotic optimization is applied in the practical system and the rich experiences are obtained in the intelligent control system.

\section{Acknowledgment}

This work was financially supported by Guangdong Province, province, the higher education innovation and strong school project (4724).

\section{Reference}

[1]CHEN Feng, GUI Wei-hua, WANG Sui-ping, SHEN De^yao, HAN Xiacrying.Modeling and Simulation of a Deep Seabed Tracked Vehicle[J]. ROBOT,2004，26（6): 510-514

[2]CHEN Feng, WANG Sui-ping, HAN X iao-ying. Self-ad 扣 stable expert fuzzy control of deep ocean m fining robot[J].JOURNAL OF CENTRAL SOUTH UNIVERSITY. 2005, 36 ( 7): 1059-1063

[3]Deepak C R, ShajahanM A, AtmanandM A. Develomental tests on the underwatermining system using fleble riser concept [A]. The 4th ISOPE OMS Proceed[C]. Hangzhou, 2001. 105 108.

[4]Handschuh R, Grebe H, Panthel J. Innovative deepceanmining concept based on flexible riser and self-ppelled mining machines [A]. The Forth ISOPE OMProceeding [C]. Hangzhou, 2001. 2529.

[5]Hu Baogang, George K.I.Mann, Raymond G.Gosine. New methodology for analytical and optimal design of fuzzy PID controller[J]. IEEE Transactions on fuzzy systems, 1999,7(5):521-538.

[6]ZOU En,LI Xiang-fei,ZHANG Tai-shang.Chaos optimization algorithm design for fuzzy neural network[J].Control Theory \&Applications.2005,8(4):578-582

[7] ZOU En,LI Xiang-fei,ZHANG Tai-shang.OPTIMAL DESIGN OF FUZZY CONTROLLER PARAMETERS BASED PN CHAOS SEARCH[J].Information and Control.2003, 32 (5):422-425

[8] ZOU En,LI Xiang-fei,ZHANG Tai-shang.Application of Fuzzy Controller Based on Chaos Optimal Design. Journal of Central South University of Technology,2004,11(1):98-101. 